\title{
Optimization of 5G Accessibility in Non Standalone and Standalone Mode
}

\author{
Adel Bouzid Belguidoum \\ LCPTS laboratory \\ Faculty of Electronics and Informatics \\ USTHB University \\ Algiers, Algeria \\ belguidoumadel@gmail.com
}

\author{
Mohamed Lamine Tounsi \\ Faculty of Electronics and Informatics \\ USTHB University \\ Algiers, Algeria \\ mltounsi@ieee.org
}

\author{
Slimane Mekaoui \\ LCPTS laboratory \\ Faculty of Electronics and Informatics \\ USTHB University \\ Algiers, Algeria \\ smekaoui@yahoo.fr
}

\begin{abstract}
In this paper, we have analyzed and developed different types of algorithms related to 5G accessibility procedures for Non Standalone (NSA) and Standalone (SA) mode. The $5 \mathrm{G}$ accessibility depends on $5 \mathrm{G}$ radio parameters of each procedure, such as contention based Random access (CBRA), contention free random access (CFRA), Radio admission control (RAC), Radio resource control (RRC) and Radio bearer reconfiguration. The random access procedure for NSA is similar to SA mode. The goal is to improve the accessibility by optimizing timers and tune the main $5 \mathrm{G}$ radio parameters related to Random access channel (RACH), RRC reconfiguration and RAC procedures.
\end{abstract}

Keywords-5G, NSA, SA, accessibility, CBRA, CFRA, RAC, $R R C$ reconfiguration, $M e N B, S g N B$, Bearer, timers.

\section{INTRODUCTION}

The fifth generation network has been already launched in 2019 [1], and the number of subscribers is increasing day by day. By $2025,5 \mathrm{G}$ networks are likely to cover one-third of the world's population [2]. This will have an impact on mobile industry and more customers will access to the provided 5G services [3]. Hence, to have a good accessibility of the services is important from a business point of view. This measurement assists the network operator with information about the accessibility provided to their clients.

Most of the $5 \mathrm{G}$ deployments so far have been NSA rollouts, it means that all these $5 \mathrm{G}$ networks have been added to an existing $4 \mathrm{G}$ infrastructure. NSA mode allows operator to launch 5G quickly and decide to use the dynamic spectrum sharing (DSS) [4]. The SA version, which does not rely on LTE, allows an operator to address massive type machine-to-machine communications (mMTC) or ultrareliable low latency (URLLC) and internet of things (IoT) [5]. It also provides network slicing functionality. Thus, some operators have decided to go straight to $5 \mathrm{G}$ new radio (NR) SA. Especially those targeting new verticals such as ultra-low latency, Edge computing, smart-grid control, industrial automation, robotics and drone control [6].

Currently with 5G NSA and SA networks, operators are focusing on the main key performance indicators (KPIs). The accessibility is considered one of the most important indicator. This KPI could be quantified from drive testing measurement for a short term optimization. Due to the coast associated from drive testing, operators prefer a long term optimization processes which tend to rely upon network statistics. The set of KPI 5G accessibility should be kept at high level to have an idea about the network access. In order to identify the main cause of KPI degradation first, we need to understand the different accessibility procedures using call flow messages. Then, classify the $5 \mathrm{G}$ parameters related to each procedure and after that require to identify in which step the failure is contributing and finally troubleshoot the problem and give the optimum solution to the issue by soft actions such as parameters tuning and recommendations. Sometimes it requires hardware actions to solve the issue immediately.

This paper is organized as follows. The 5G accessibility procedures and parameters are given in Section II, and 5G accessibility optimization and algorithms are mentioned in Section III and some numerical results are presented in Section IV. A Conclusion is drawn in Section V.

\section{5G ACCESSIBILITY AND PROCEDURES}

5G accessibility support two different modes NSA and SA. The NSA accessibility contains eight (8) procedures which cover the events from the UE that sends the first transmission on a LTE Master eNB (MeNB) cell until the $5 \mathrm{G}$ random access channel procedure $(\mathrm{RACH})$ has been completed on the $5 \mathrm{G}$ secondary gNB ( $\mathrm{SgNB})$ cell. On the other hand the SA accessibility is limited into three (3) procedures. It starts from the $\mathrm{UE}$ is in Radio resource control (RRC) idle mode on a $5 \mathrm{G}$ cell until the $5 \mathrm{G}$ radio bearer is setup on the $5 \mathrm{G}$ cell. The $5 \mathrm{G}$ radio access network (RAN) and the 5G Core Network $(\mathrm{CN})$ are involved in this procedure; the LTE part of the network is not involved at all.

\section{A. NSA and SA mode}

According to 3GPP [7], "option 2" and "option 3x" deployments are supported for SA and NSA mode. Both options can be enabled concurrently in the same cell, and idle mode parameters are used to determine if a 5G-capable UE camps on a $4 \mathrm{G}$ cell (NSA mode, option $3 \mathrm{x}$ ) or on a $5 \mathrm{G}$ cell (SA mode, option 2). In option 3x, a LTE cell is used as "anchor cell" where the RRC connection is first established via the LTE radio link and afterwards a $5 \mathrm{G}$ user plane connection is added on top of the LTE user plane connection. This arrangement is referred to as EUTRAN NR Dual Connectivity (EN-DC). The $4 \mathrm{G}$ core network (the EPC) continues to be used with minor modifications in the MME and in the SGW [8]. In option 2, there is no need to have LTE coverage. The 5G RRC connection is directly setup on the 5G BTS (the gNB), which is connected to the $5 \mathrm{G}$ core network $(5 \mathrm{G}-\mathrm{CN})$ via the $\mathrm{NG}-\mathrm{C}$ interface. IntraRAT handovers can take place via the $\mathrm{Xn}$ interface and inter-RAT handover to $4 \mathrm{G}$ can take place via the $\mathrm{X} 2$ interface. With this option, the UE will not have simultaneous radio connections on both $4 \mathrm{G}$ and $5 \mathrm{G}$ radio interfaces. As describe in figure 1 , the centralized unit (CU) 
and distributed unit (DU) are virtual entities located inside the same box connected with a virtual F1 interface [9].

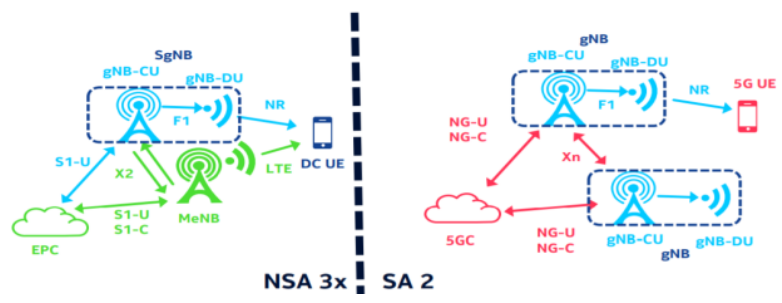

Fig. 1. NSA Option 3x and SA option 2 architecture

\section{B. NSA and SA accessibility procedures}

The various steps of NSA and SA accessibility procedures are described in Table I and Table II.

For NSA accessibility from step 1 to step 3 a dual capable user equipment (DC-UE) is still in LTE cell and has obtained the signalling radio resources (SRB0, SRB1 and SRB2), data radio bearer resource (DRB) and eRAB has been established. In step 4, a DC-UE starts to measure 5G radio signal and prepares for $\mathrm{SgNB}$ addition in step5. After a successful admission control and SgNB addition, we will have a radio resource control (RRC) reconfiguration procedure in step 6 . In step7, the eNB informs the gNB about the exact place in the PDCP data stream where gNB takes responsibility (the Sequence Number is transferred) and the eNB informs the EPC that the user plane connection between RAN and EPC should be switched from eNB to gNB. Finally, in step 8 a DC-UE will access to $5 \mathrm{G}$ cell using a RACH procedure.

For SA accessibility from step A to step C, UE start with $\mathrm{RACH}$ procedure, then RRC establishment and will finish by DRB establishment. In terms of latency the SA access is faster than NSA access.

TABLE I. NSA ACCESSIBILITY PROCEDURES

\begin{tabular}{|c|l|}
\hline Procedures & \multicolumn{1}{|c|}{ NSA Accessibility-content } \\
\hline $\mathbf{1}$ & EN-DC capable UEs will camp on the LTE cell \\
\hline $\mathbf{2}$ & LTE RRC connection is completed \\
\hline $\mathbf{3}$ & eRAB and Data Radio bearer setup on LTE cell \\
\hline $\mathbf{4}$ & UE measures good enough 5G radio signal \\
\hline $\mathbf{5}$ & SgNB addition and 5G radio admission control \\
\hline $\mathbf{6}$ & 5G RRC reconfiguration \\
\hline $\mathbf{7}$ & 5G status transfer by switching user plane to gNB \\
\hline $\mathbf{8}$ & Successful 5G RACH procedure \\
\hline
\end{tabular}

TABLE II. SA ACCESSIBILITY PROCEDURES

\begin{tabular}{|l|l|}
\hline Procedures & SA Accessibility-content \\
\hline
\end{tabular}

\begin{tabular}{|c|l|}
\hline Procedures & \multicolumn{1}{|c|}{ SA Accessibility-content } \\
\hline A & Successful 5G RACH procedure \\
\hline B & 5G RRC connection is completed \\
\hline C & Data radio bearer establishement in 5G cell \\
\hline
\end{tabular}

Figure 2 shows the NSA accessibility call flow from $5 \mathrm{G}$ point of view. The formula of 5G NSA accessibility success rate $(\mathrm{SR})$ is defined by the following equation (1):

5G NSA accessibility $\mathrm{SR}=\mathrm{SgNB}$ addition $\mathrm{SR} * \mathrm{RRC}$ reconfiguration $\mathrm{SR} * \mathrm{RACH} \mathrm{SR}$

where, the $\mathrm{SgNB}$ addition SR is given by equation (2)

$$
\text { SgNB addition } \mathrm{SR}=\frac{S g N B \text { addition Acknowledge }}{S g N B \text { addition request }}
$$

the $\mathrm{RRC}$ reconfiguration SR is defined by equation (3)

$\mathrm{RRC}$ reconfiguration $\mathrm{SR}=\frac{R R C \text { reconf } \text { iguration complete }}{R R C \text { reconf iguration }}$

and RACH SR is calculated as per equation (4)

$$
\text { RACH SR }=\frac{M S G 3 \text { contention } \text { based }}{\text { RACH Attempt }}
$$

Assume that SN Status transfer procedure is successful, then RRC reconfiguration complete message 6 (MSG 6) is equal to $\mathrm{RACH}$ attempt message 7 (MSG 7), in this case the equation (1) will be as follow:

$$
5 \text { G NSA accessibility SR }=\frac{M S G 3 \text { contention } \text { based }}{S g N B \text { addition request }}
$$

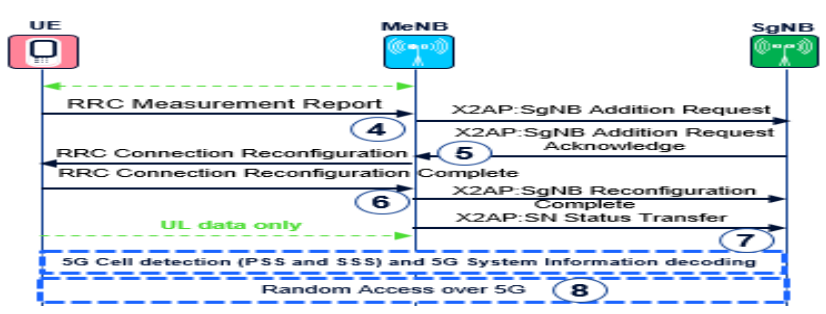

Fig. 2. 5G NSA accessibility call flow

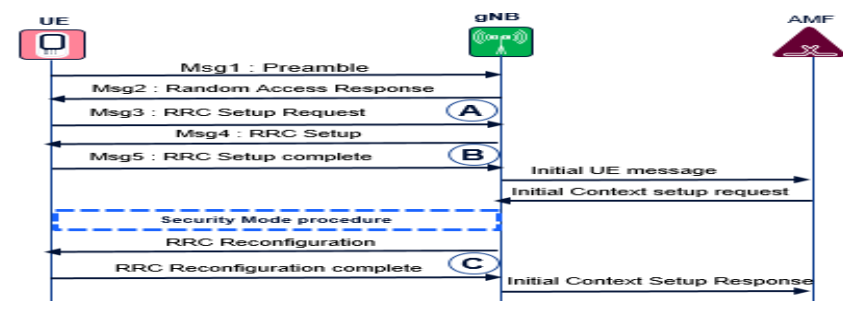

Fig. 3. 5G SA accessibility call flow

Figure 3 presents the 5G SA accessibility call flow. The formulas of this KPI is defined by the equation (6) below:

5G SA accessibility SR $=$ RACH SR * 5G RRC connection setup $\mathrm{SR} * \mathrm{RRC}$ reconfiguration $\mathrm{SR}$ 
Both KPIs RACH SR and RRC reconfiguration SR for SA mode are similar to NSA mode defined in equations (3) and (4). A new KPI is 5G RRC connection (conn) setup SR which is defined by the following equation (7):

$$
5 \mathrm{G} \text { RRC conn setup } \mathrm{SR}=\frac{R R C \text { setup complete }}{R R C \text { conn request }}
$$

Finally, the SA accessibility SR will be defined as follow:

$5 \mathrm{G}$ SA accessibility SR $=\frac{R R C \text { reconfiguration complete }}{\text { Total } \mathrm{RACH} \text { preambles }}$

In order to improve the SR of $5 \mathrm{G}$ NSA and SA accessibility defined in equations (5) and (8), we need to focus on RACH procedure, radio admission control (RAC) procedure and RRC reconfiguration procedure.

\section{Random Access procedure}

The main purpose of RACH procedure is to obtain the resource for message 3 (MSG 3) and initial physical layer (L1) synchronization for timing and power. It is needed for both mode NSA and SA. In 5G, we have two different procedures for random access the same as in LTE. The first procedure is called contention based random access (CBRA) and the second is contention free random access (CFRA). The CBRA is used during an initial access, RRC connection re-establishment, loss of uplink (UL) synchronization, beam recovery, transition from RRC inactive state, time alignment procedure and also used when CFRA cannot be performed. The CFRA is mainly used during an SgNB addition in NSA mode and handover procedures. As describe in figure 3 below, in the CBRA procedure a UE selects the preamble message 1 (MSG1) but for CFRA procedure, the gNB will assign the dedicated preamble message zero (MSG 0). The CFRA is faster and more reliable than CBRA procedure.
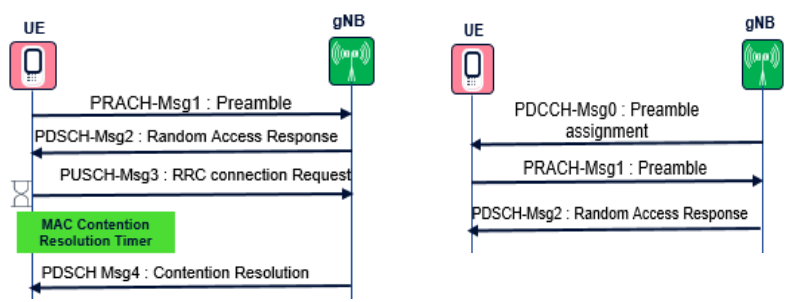

Fig.4. 5G CBRA (LEFT) AND CRFA (RIGHT) PROCEDURES

The optimization of RACH procedure in NSA mode is the same as SA mode. Some of 5G RACH parameters defined in Table III are configured during a radio planning such as root sequence Index parameter. These root sequences should be allocated with a re-use pattern which avoids neighboring cells using the same root sequences.

\section{TABLE III. 5G RACH RADIO PLANNING PARAMETERS}

\begin{tabular}{|c|l|}
\hline Parameters & Description of 5G RACH- Radio planning \\
\hline Root Sequence Index & $\begin{array}{l}\text { used to generate the set of PRACH } \\
\text { preambles }\end{array}$ \\
\hline Msg1 Frequency Start & PRACH position in the frequency domain \\
\hline $\begin{array}{c}\text { RACH Configuration } \\
\text { Index }\end{array}$ & $\begin{array}{l}\text { Define the PRACH position in the time } \\
\text { domain and determinate the preamble format }\end{array}$ \\
\hline
\end{tabular}

\begin{tabular}{|c|l|}
\hline Parameters & Description of 5G RACH- Radio planning \\
\hline $\begin{array}{c}\text { Zero Correlation Zone } \\
\text { Config }\end{array}$ & Define the expected cell range \\
\hline
\end{tabular}

The parameter Zero correlation config defined in Table III will help too much to improve $\mathrm{RACH}$ performance in rural area. The RACH procedure will fail if the UE is located/) outside the planned cell range.

Based on 3GPP Release 15 [10], a UE transmit RACH Preamble (MSG1) using the following equation (9):

Preamble received Target power $=$ Initialpreamblepower

$$
+(\text { preamble Transmision counter }-1) * \text { PowerRampingStep }
$$

The parameter PreambleTransMax listed in TABLE IV defines the maximum number of Random access preamble transmission [11]. If we reach the maximum preamble transmission without receiving MSG2 random access response during a period of time defined by the timer RandomResponseWindow, a UE will fail to access to the cell and will reselect another cell. CFRA and CBRA procedures will also fail. Table IV shows the parameters related to different messages of CFRA and CBRA procedures.

TABLE IV. 5G PARAMETERS FOR CFRA AND CBRA PROCEDURES

\begin{tabular}{|c|c|c|}
\hline 5G NSA/SA & \multicolumn{2}{|c|}{ CFRA and CBRA Flow messages } \\
\hline Parameter & Message & Definition \\
\hline Initialpreamblepower & MSG 1 & Initial power for RA preamble \\
\hline PowerRampingStep & MSG 1 & Power increment step size \\
\hline PreambleTransMax & MSG 1 & Max number of RA Trans \\
\hline RandomResponseWindow & MSG 2 & Window size for RAR \\
\hline maxHarqMsg3Tx & MSG 3 & Max number of HARQ Trans \\
\hline raContResolutionTmr & MSG 4 & Contention resolution timer \\
\hline
\end{tabular}

\section{D. $5 G$ radio admission control procedures}

The capacity utilization and congestion must be measured during the busy period. A radio admission control (RAC) is used to prevent a congestion on the network [12]. If the maximum cell capacity is reached then the RAC will reject a new admission request. The admission control is also performed when a new connection is established and when there is an incoming handover. The admission control performance must be analyzed for both units CU and DU.

The radio admission control in $\mathrm{CU}$ is performed based on the number of active UE operating in NSA mode during $\mathrm{SgNB}$ addition procedure or in SA mode during the RRC connection establishment procedure. The distributed unit will check only if there is enough capacity for physical uplink control channel $(\mathrm{PUCCH})$. The PUCCH congestion is therefore only caused by too many channel state information (CSI) reports. This issue can have an impact on admission control DU for NSA and SA modes. Figure 5 shows the admission control for NSA mode during $\mathrm{SgNB}$ addition procedure. 
Fig. 5. Admission control during SgNB addition procedure

Figure 6 presents the admission control for SA mode during RRC connection establishment procedure.

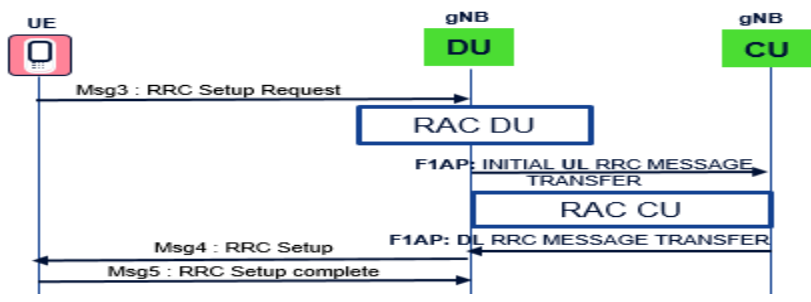

Fig. 6. Admission control during RRC connection procedure

\section{E. $5 G$ radio ressource control reconfiguration procedure}

The 5G RRC reconfiguration procedure [13], can be used for NSA mode after the MeNB has received the SgNB Addition Request Acknowledge message on X2 (containing information to $\mathrm{UE}$ about the $5 \mathrm{G}$ radio configuration), the MeNB forwards this information to UE (in RRC Connection Reconfiguration message), waits for acknowledgement and then forwards the acknowledgment to the $\mathrm{SgNB}$ as shown in figure 7 . There are two timers which handle this procedure from both sides $4 \mathrm{G}$ and 5G. RRC Guard timer (rrcGuardTimer) is running in $4 \mathrm{G}$ and timer dual connectivity over all (tDCoverall) is controlling $5 \mathrm{G}$ side.

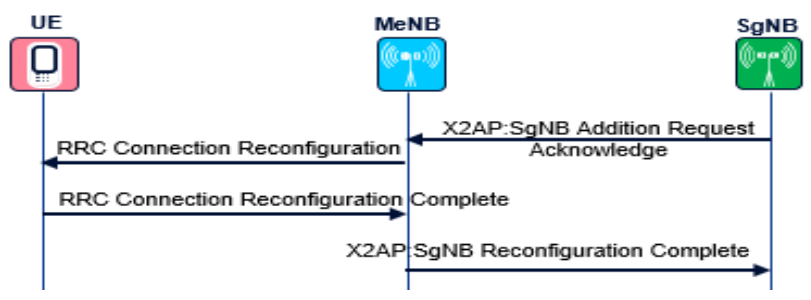

Fig. 7. RRC reconfiguration procedure for NSA mode

The 5G RRC reconfiguration procedure can be used also in SA mode during radio bearer establishment.

\section{OPTIMIZATION OF 5G ACCESSIBILITY}

The optimization of $5 \mathrm{G}$ accessibility KPI is defined based on a certain threshold which is almost close to hundred percent $(100 \%)$. Some operators fix a threshold at $99.8 \%$. The measurement need to be evaluated daily and on the busy hour. Both KPIs 5G NSA accessibility SR and 5G SA accessibility SR mentioned in Section II include in their formulas the KPI RACH SR. This KPI is considered as one of the main important $5 \mathrm{G}$ indicator and its optimization is

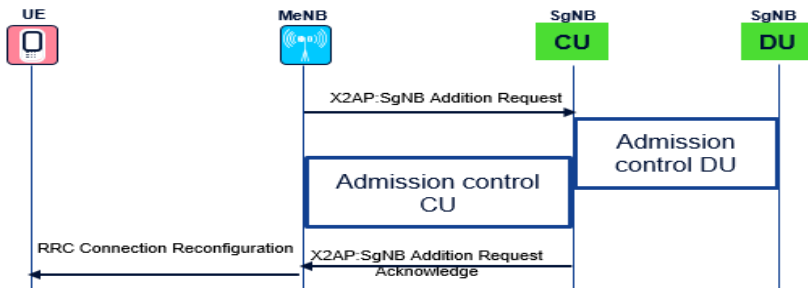

difficult and need to have a very good knowledge in the air interface [14], parameters and flow messages related to $\mathrm{RACH}$ procedure in order to understand the root cause of $\mathrm{RACH}$ failure. These failure could be related to bad radio planning, wrong parameters setting, poor coverage, overshooting, etc.

We have developed a new process to go through the main cause of degradation and propose the optimum solution related to each issue identified during the accessibility KPI analysis. We have some quick wins actions which can help us to improve the performance. These actions are timers and $5 \mathrm{G}$ radio parameters defined for each procedure. Our algorithm check all the NSA and SA procedures defined earlier in section II. If the KPI of the first procedure is below a certain threshold, it will propose a best soft action, otherwise, it will go to the next procedure and compare its KPI to another threshold and so on till the last test. This algorithm links all $5 \mathrm{G}$ radio parameters to the adequate procedure. At the end it will be very easy for user to check the current parameter value and can recommend the optimum value.

Figure 8 presents an optimization algorithm of 5G KPI RACH success rate for NSA and SA Mode. From the root cause selection, we can determine the appropriate action to solve the issue.

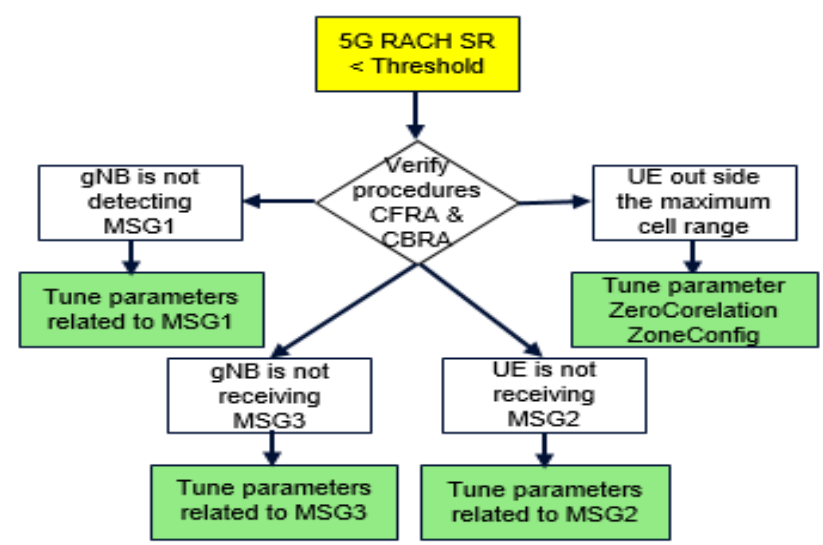

Fig. 8. Optimization of 5G RACH Success rate

Figure 9 illustrates an optimization algorithm of 5G KPI Radio admission control rejection ratio for NSA and SA Mode. The rejection is handled for both units DU and CU. For the DU, we have validated the same action for NSA and SA Mode because of lack of PUCCH resources in the air interface. 
Fig. 9. Optimization of $5 \mathrm{G}$ radio admission control rejection ratio

Figure 10 shows an optimization algorithm of 5G KPI RRC reconfiguration Success rate for NSA and SA Mode. The best action is tuning different timers. RRC Guard Timer can be used in LTE and in 5G SA mode, but Dual connectivity timer is used only for NSA mode.

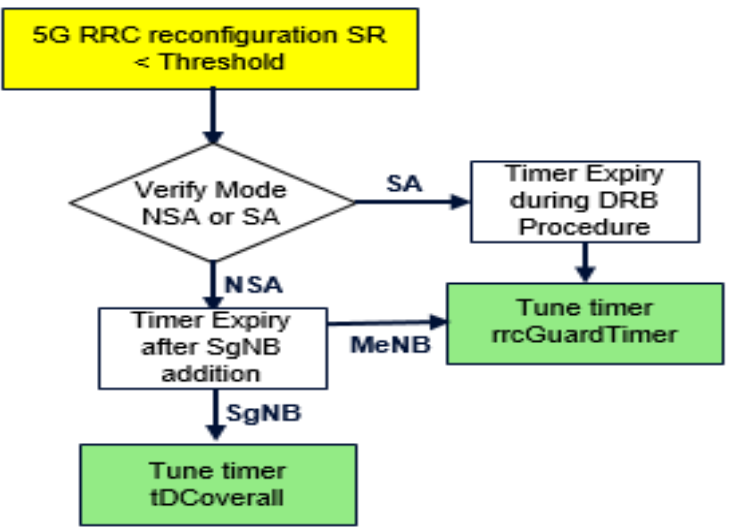

Fig. 10. Optimization of 5G RRC reconfiguration Success rate

\section{RESULTS AND DISCUSSION}

The algorithm of $5 \mathrm{G}$ accessibility is based on measurement KPIs. The calculation is based on the fine tuning of $5 \mathrm{G}$ radio parameters and timer optimization. The obtained results are presented for different KPIs related to NSA accessibility such as random access success ratio, radio admission control rejection ratio and RRC reconfiguration success ratio and are showing the impact of radio parameters.

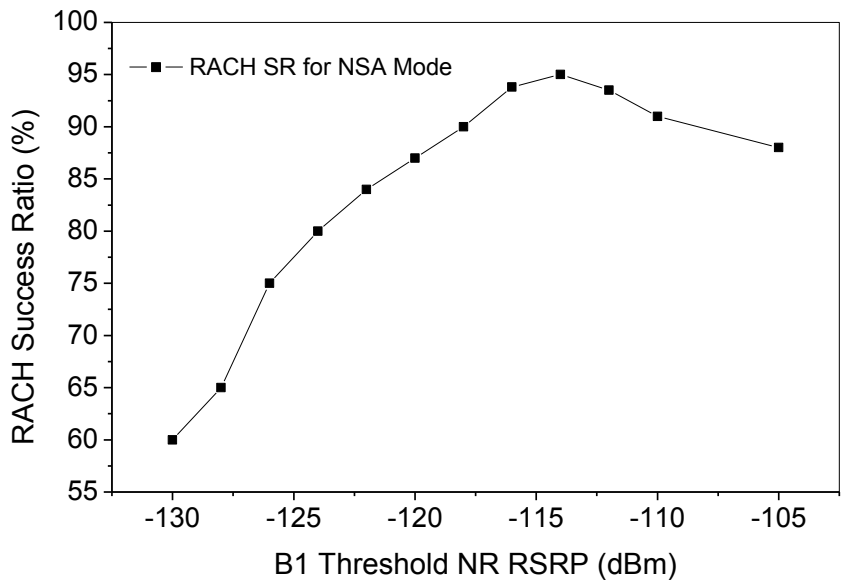

Fig. 11. Impact of parameter "B1ThresholdNRRSRP” on KPI RACH SR

Figure 11 shows the impact of radio parameter "B1 Threshold NR RSRP" on the KPI RACH Success ratio. The results show an improvement in RACH SR for NSA Mode when the parameter value is increased. A stronger and more stable reference signal received power (RSRP) will help for a successful of RACH procedure. The recommended value of

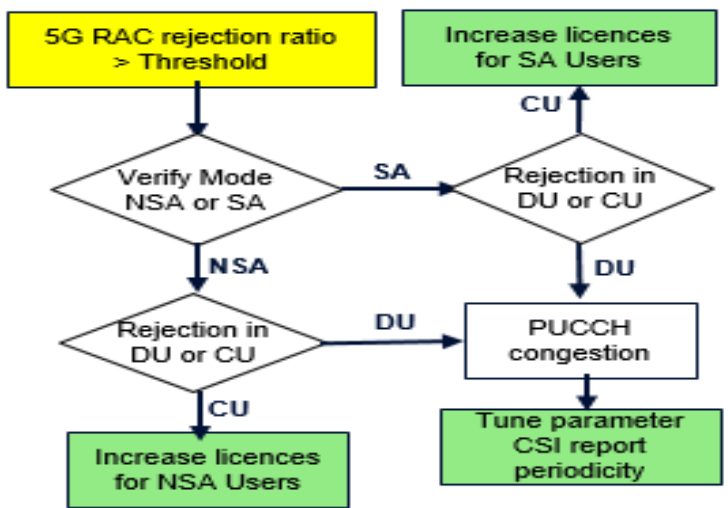

this parameter is $-114 \mathrm{dBm}$ where we have obtained $95 \%$ on RACH SR which is considered as a very good improvement on NSA accessibility success rate.

As we have seen in Section III, the optimization of RACH SR and one of the critical issue is when the UE is outside the maximum cell range. This will degrade the $\mathrm{RACH}$ performance. The idea is to optimize the cell range in order to improve RACH SR by increasing the value of the parameter "Zero Correlation Zone config". Figure 12 shows the impact of this parameter on the cell range in meters for a different value of numerology $(\mu)$ [15]. In centimeter wave the cell range is higher than millimeter wave. The recommended value will depend on the network radio planning. If small cells are deployed, it is better to decrease the parameter "Zero Correlation Zone config" in order to avoid a PRACH interference due to bad root sequence index planning.

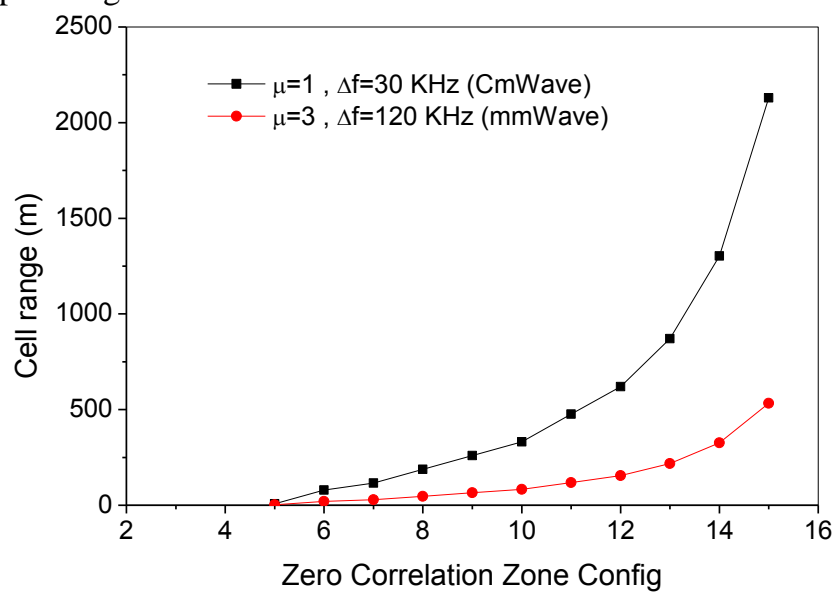

Fig. 12. Impact of parameter "ZeroCorrelationZoneConfig" on cell range

Figure 13 presents the impact of parameter "CSI report periodicity" on both KPIs radio admission control rejection ratio and the number of $5 \mathrm{G}$ users. A UE report Channel state information (CSI) each period on the physical control channel (PUCCH). This can lead to admission control rejection and $\mathrm{PUCCH}$ congestion when the CSI report is high. In order to decrease the number of CSI report, it recommended to increase the periodicity as you can see in figure 13, the RAC rejection ratio is equal to zero when the CSI periodicity is set to high value 320 slots, the maximum number of $5 \mathrm{G}$ users reached 160 users per cell and when the 
CSI periodicity is set to low value 20 Slots the maximum capacity is reached and RAC rejection ratio is very high at $90 \%$ and the maximum number of users is limited at 10 Users.

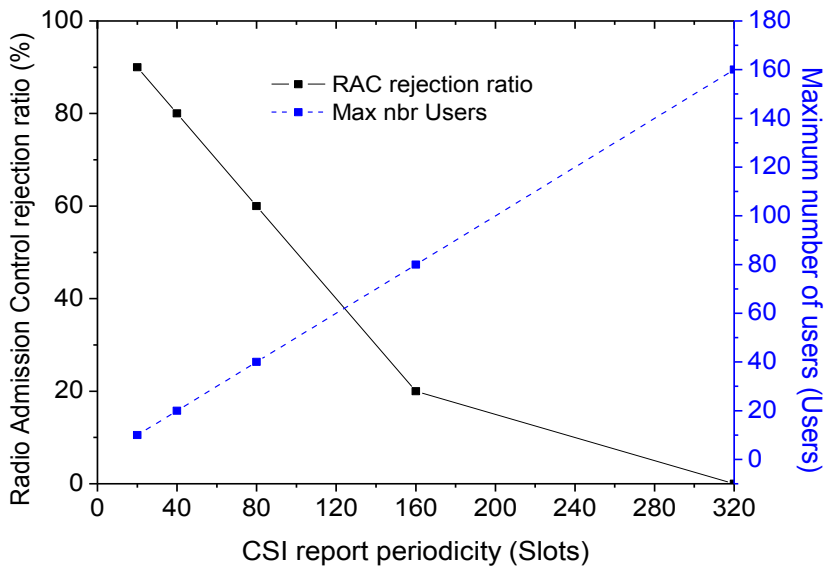

Fig.13. Impact of parameter "CSI report periodicity" on KPIs Radio admission control rejection ratio and Number of Users

Figure 14 illustrates the effect of timer "RRC Guard timer" on the KPI RRC reconfiguration success rate. When the value of timer is set too low at $100 \mathrm{~ms}$ the RRC reconfiguration SR was bad and below the target $80 \%$, by increasing this timer the KPI improves too much and have reached $100 \%$ for a value of timer equal to $4000 \mathrm{~ms}$. Setting this timer too high and more than $6000 \mathrm{~ms}$, can have a negative impact on the $5 \mathrm{G}$ latency. There is a tradeoff between $5 \mathrm{G}$ accessibility and latency.

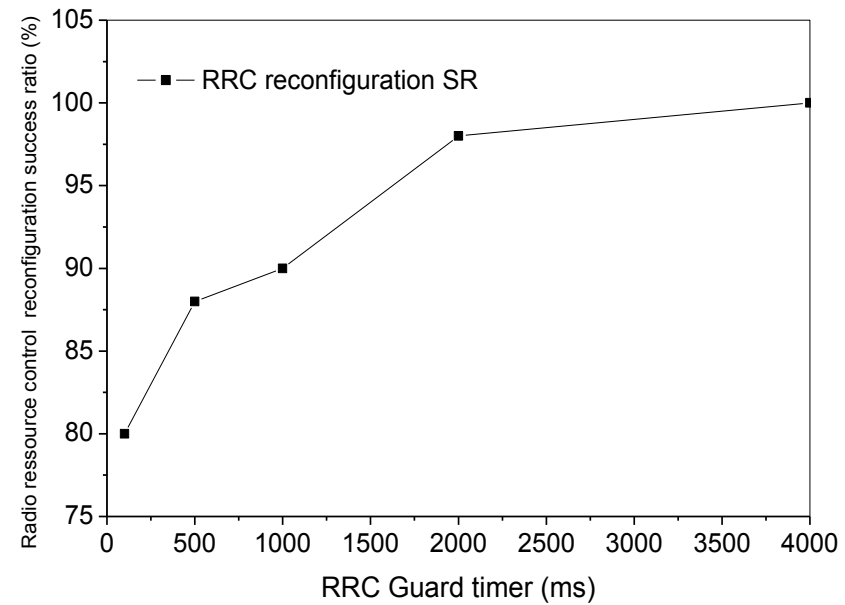

Fig. 14. Impact of Timer " RRC guard Timer" on KPI RRC reconfiguration $\mathrm{SR}$

\section{CONCLUSION}

In this paper, we have introduced a new approach of optimization of $5 \mathrm{G}$ accessibility for NSA and SA mode. We have started by analyzing KPIs formulas related to each procedure (CBRA, CFRA, RAC and RRC reconfiguration), then we have developed a different type of algorithms and based on degradation, we have proposed a parameter tuning in order to improve the $5 \mathrm{G}$ accessibility. We have evaluated the impact of radio parameters on KPI 5G random access success ratio which is considered as one of the most important indicator for NSA and SA accessibility. We also have evaluated the impact of timers on Radio admission control rejection ratio and RRC reconfiguration success rate. We conclude that the SA accessibility is more robust than NSA accessibility since it has a few procedures compared to NSA procedures. It will be very easy to reach the target value $99.5 \%$ and in terms of capacity it is promising that SA users will access to the network and release the signaling resources quickly.

\section{REFERENCES}

[1] Wikipedia, List of 5G NR networks, "Commercial deployments" https://en.wikipedia.org/wiki/List_of_5G_NR_networks/, April 2020.

[2] GSMA 5G Global Launches \& Statistics, , " The Global Rollout of $5 \mathrm{G}$ ”

https://www.gsma.com/futurenetworks/ip_services/understanding$5 \mathrm{~g} / 5 \mathrm{~g}$-innovation/, May 2020

[3] Digitaltrends, Mark Jansen and Kerry Wan,“ Every 5G phone announced so far so you can get a faster internet connection," https://www.digitaltrends.com/mobile/5g-capable-phones/, July 2020.

[4] S. Krishna Sharma, T. Endeshaw Bogale, L. Bao Le, S. Chatzinotas, X. Wang, B. Otters, "Dynamic Spectrum Sharing in 5G Wireless Networks With Full-Duplex Technology, IEEE (2017) 674 - 707,doi: 10.1109/COMST.2017.2773628.

[5] Ejaz et al., "Internet of Things (IoT) in 5G Wireless Communications," in IEEE Access, vol. 4, pp. 10310-10314, 2016.

[6] Iot Business News, "T-Mobile Launches World's First Nationwide Standalone 5G Network, Taking 5G Next-Level"

https://iotbusinessnews.com/2020/08/05/69855-t-mobile-launchesworlds-first-nationwide-standalone-5g-network/, August 2020.

[7] 5G Americas, "5G-Evolution-3GPP-R16-R17-FINAL”, pp. 35-36, January, 2020.

[8] 3GPP TS 23.214 version 16.0.0 Release 16, Architecture enhancements for control and user plane separation of EPC nodes, pp. 8-19, June 2019.

[9] 3GPP TS 38.473 version 16.2.0 Release 16, NG-RAN, F1 application protocol (F1AP), pp. 13-70, July 2020.

[10] 3GPP TS 38.321 version 15.5.0 Release 15, 5G; NR; Medium Access Control (MAC) protocol specification, pp. 13-70, May 2019.

[11] Evolved Universal Terrestrial Radio Access (E-UTRA); Physical Channels and Modulation, 3GPP TS 36.211 V10.4.0, 2012.

[12] S. B. Deshmukh and V. V. Deshmukh, "Call admission control in cellular network," International Journal of Advanced Research in Electrical, Electronics and Instrumentation Engineering, vol. 2, no. 4, pp. 1388-1391, 2013.

[13] 3GPP TS 38.331 version 15.3.0 Release 15, 5G; NR; Radio Resource Control (RRC); Protocol specification, pp. 40-48, October 2010.

[14] 3GPP TR 38.802 V14.2.0, "Study on New Radio Access Technology Physical Layer Aspects,", 2017.

[15] A.B Belguidoum, M.L Tounsi, S. Mekaoui, "Analysis of 5G Radio Parameters and their Impact on the Peak Data Rate," in IEEE, pp. 1-6, December, 2019.

Creative Commons Attribution License 4.0 (Attribution 4.0 International, CC BY 4.0)

This article is published under the terms of the Creative Commons Attribution License 4.0 https://creativecommons.org/licenses/by/4.0/deed.en_US 